03

\title{
Вопросы термодинамики турбулентной конвекции
}

\author{
() А.М. Кригель
}

Санкт-Петербургский фрилиал „Издательство „Наука“, 199034 Санкт-Петербург, Россия

e-mail: amkrigel@gmail.com

(Поступило в Редакцию 11 ноября 2015 г.)

Применяя уравнение производства энтропии к идеальному однокомпонентному турбулентному газу, в допущении политропности флуктуаций, а также теорему И. Пригожина, найдены термодинамические свойства стационарного потока в поле силы тяжести. Рассмотрены приложения к задачам динамики приземного слоя атмосферы и свободной конвекции.

\section{Введение}

На уроках физики в школе ставится вопрос - что произойдет с воздухом при его нагревании? Ответ известен - газ при нагревании расширяется, его плотность падает и под действием силы Архимеда он устремится вверх. Это действительно так, если давление газа при его нагревании не изменится существенно. Однако, считая газ идеальным, из уравнения Менделеева-Клапейрона $[1,2]$ следует, что относительные флуктуации давления, температуры и плотности связаны друг с другом соотношением

$$
\frac{\partial P}{P}=\frac{\partial \rho}{\rho}+\frac{\partial T}{T},
$$

где $P$ - давление, $\rho$ - плотность, $T-$ абсолютная температура. Рассмотрим политропный процесс, который характеризуется тем, что удельная теплоемкость газа $C$ принимается постоянной. Тогда, как известно, выполняется уравнение Пуассона [1,2]

$$
\frac{\partial P}{P}=\varkappa \frac{\partial \rho}{\rho}=\frac{\varkappa}{(\varkappa-1)} \frac{\partial T}{T}
$$

где

$$
\varkappa=\frac{C_{p}-C}{C_{v}-C}
$$

- показатель политропы, $C_{p}$ - удельная теплоемкость в изобарном процессе, $C_{v}-$ удельная теплоемкость в изохорном процессе.

Чтобы ответить на школьный вопрос, надо знать удельную теплоемкость атмосферы, но она заранее не известна, поскольку определяется типом термодинамического процесса. Так, в теории звука допускается [3], что малые возмущения можно считать адиабатическими $(C=0)$, тогда из (3) следует, что $\varkappa=\gamma=C_{p} / C_{v}>1$. Но тогда из (2) вытекает, что плотность газа при нагревании увеличивается, что прямо противоположно тому, что имеет место при конвекции. Видимо термодинамика звуковых и конвективных возмущений различна, и это не удивительно, поскольку конвекция в отличие от звука явно не адиабатический процесс.

Рассмотрим неравновесный процесс в однокомпонетном турбулентном газе. Запишем уравнение эволюции энтропии в виде [4]

$$
\frac{\partial}{\partial t}(\rho \eta)+\frac{\partial}{\partial x_{i}}\left[\left(\rho v_{i}+S_{i}\right) \eta+J_{i}\right]=\Sigma_{m}+\Sigma_{t},
$$

где $\eta$ - энтропия, отнесенная к единице массы, $v_{i}-$ вектор скорости упорядоченного движения, $\Sigma_{m}$ и $\Sigma_{t}-$ скорости производства энтропии в единице объема, определяемые молекулярными и турбулентными флуктуациями соответственно,

$$
S_{i}=\overline{\rho^{\prime} v_{i}^{\prime}}
$$

- вектор плотности турбулентного потока вещества (турбулентный импульс), (черта сверху означает осреднение, штрих - отклонение от среднего),

$$
J_{i}=\frac{H_{i}+C_{p} T S_{i}+Q_{i}+I_{i}}{T}
$$

— вектор плотности турбулентного потока энтропии, включающего в себя

$$
H_{i}=C_{p}\left(\rho \overline{v_{i}^{\prime} T^{\prime}}+\overline{\rho^{\prime} v_{i}^{\prime} T^{\prime}}\right)
$$

- плотность турбулентного потока тепла, $Q_{i}$ плотность потока тепла, порожденного молекулярными флуктуациями, $I_{i}$ - плотность турбулентного потока энергии турбулентности, по повторяющимся индексам, как обычно, подразумевается суммирование.

Для политропных флуктуаций, учитывая (2), (5) и пренебрегая тройными корреляциями, выражение (7) преобразуется к виду

$$
H_{i}=(\varkappa-1) C_{p} T S_{i} .
$$

Из этого выражения вытекает, что турбулентный поток тепла и турбулентный импульс пропорциональны друг другу и, следовательно, определяются одним и тем же неравновесным процессом.

Второе начало термодинамики, как известно, утверждает что производство энтропии неотрицательно, что имеет место, если правая часть уравнения (4) сводится к сумме квадратичных форм [2]. Следствием этого утверждения является то, что плотность турбулентного 
потока тепла в присутствии внешнего поля связана с термодинамической силой, порождающей этот поток, кинетическим соотношением [4]

$$
H_{i}=\rho C_{p} K\left(\Gamma_{i}-\frac{\partial T}{\partial x_{i}}\right)
$$

где $K$ - коэффициент турбулентной теплопроводности,

$$
\Gamma_{i}=\frac{g_{i}}{\beta C_{p}}
$$

- „равновесный градиент температуры“, $g_{i}$ - ускорение, вызванное действием силы тяжести, или иной внешней массовой силы,

$$
\beta=\frac{\gamma-1}{\gamma(\varkappa-1)}+\varkappa-\frac{1}{\gamma}
$$

Поскольку теплоемкость газа зависит от термодинамического процесса, значение $\beta$ не может быть задано априорно. В случае отсутствия внешнего поля поток тепла, как известно, следует закону Фурье [2,3], к которому мы придем, если в (9) принять $\Gamma_{i}=0$. В процессах лабораторного масштаба поток тепла, вызванный действием массовой силы, как правило, пренебрежимо мал. Но в задачах физики атмосферы и в астрофизике, в которых пренебрежение этим эффектом становится недопустимым, принимается (без убедительного доказательства), что условие термического равновесия совпадает с условием возникновения конвекции [3], к которому выражение (10) сводится при $\beta=1$ („адиабатический градиент температуры“). Этот вывод опирается на допущение об адиабатичности малых возмущений идеального газа. Но тогда из (8) вытекает, что турбулентный поток тепла и поток вещества направлены в одну сторону, что противоположно тому, что имеет место при конвекции. Чтобы выйти из этого затруднения, пренебрегая логикой, полагают (в рамках так называемого „приближения Буссинеска“" $[3,5])$, что в выражении (8) газ ведет себя как несжимаемая жидкость $\left(C=C_{p}\right)$, в то время как в теории турбулентной теплопроводности возмущения полагаются адиабатическими $(C=0)$. Аэрологические наблюдения [6] не оправдывают применение теории Буссинеска к свободной атмосфере. При таком положении вещей мы вынуждены признать, что принятые в настоящее время теория турбулентной теплопроводности и теория конвекции в газе страдают серьезными противоречиями.

Решение проблемы может быть найдено в случае стационарного неравновесного процесса. Согласно теореме Пригожина [7], неравновесный процесс при неизменных внешних условиях, препятствующих достижения равновесного состояния, становится стационарным при минимальном производстве энтропии. Используя этот принцип, можно найти удельную теплоемкость газа, что и даст теоретическую основу для решения задачи о турбулентной теплопроводности.

\section{Перенос тепла при постоянном коэффициенте теплопроводности}

Рассмотрим слабый неравновесный процесс, для которого основные принципы термодинамики сохраняют силу. Скорость производства энтропии, вызванного действием турбулентной теплопроводности вблизи состояния термодинамического равновесия, имеет вид [4]

$$
\Sigma_{t}=\frac{S_{i}}{T}\left(g_{i}-\beta C_{p} \frac{\partial T}{\partial x_{i}}\right)=\frac{\beta H_{i}^{2}}{\rho C_{p} T^{2} K(\varkappa-1)} \geq 0 .
$$

Рассмотрим процесс стационарного переноса тепла в поле силы тяжести, при котором коэффициент турбулентной теплопроводности величина положительная, постоянная, не зависящая ни от потока тепла, ни от удельной теплоемкости газа. Будем искать минимум выражения (12) при неизменной плотности турбулентного потока энтропии. Тогда, учитывая (6), (8)-(11) и рассматривая выражение (12) как функцию $\alpha=C / C_{v}$, нетрудно найти, что производство энтропии достигает минимума при $\alpha=-\gamma, \varkappa=2 \gamma(\gamma+1)$. Тогда для земной атмосферы, состоящей в основном из двухатомных газов, для которых $\gamma=7 / 5$, получаем, что $\alpha=-1.40$, $\varkappa=1.17, \beta=-2.33$. Из того что $\alpha<0$, следует, что газ в этом примере работает как тепловой насос, а поскольку $x>1$, его плотность при нагревании растет.

\section{Свободная конвекция}

Рассмотрим стационарную свободную конвекцию газа в поле силы тяжести. Запишем уравнение баланса кинетической энергии турбулентного движения [5] в виде

$$
\frac{\partial\left(\rho b^{2}\right)}{\partial t}=\rho K_{v}\left(\frac{\partial v_{1}}{\partial x_{3}}\right)^{2}+g_{3} S_{3}-\frac{\partial I_{i}}{\partial x_{i}}-\varepsilon,
$$

где

$$
\rho b^{2}=\frac{\left(\rho \overline{v_{i}^{\prime} v_{i}^{\prime}}+\overline{\rho^{\prime} v_{i}^{\prime} v_{i}^{\prime}}\right)}{2},
$$

- плотность кинетической энергии турбулентности, $b$ - „скорость турбулентных флуктуаций“, $K_{v}-$ коэффициент турбулентной вязкости, $\varepsilon-$ скорость диссипации энергии турбулентности. Направим ось $x_{3}$ вертикально вверх, а вектор скорости упорядоченного течения горизонтально по оси $x_{1}$. Примем основные принципы теории турбулентности, основанной на работах А.Н. Колмогорова и А.М. Обухова [5], в которой полагается, что в изотропной среде имеет место

$$
\begin{gathered}
K_{v}=l b, \\
\varepsilon=\rho \frac{\sigma b^{3}}{l},
\end{gathered}
$$

где $l-$ масштаб турбулентности, $\sigma \sim 0.046-$ константа диссипации, а дивергенцией потока кинетической энергии турбулентности $I_{i}$ можно пренебречь. В случае 
свободной конвекции в правой части (13) можно также пренебречь и механизмом генерации турбулентности сдвигом скорости упорядоченного потока по сравнению с работой силы Архимеда. Тогда из (13)-(16) получаем, что

$$
b^{3}=\frac{g_{3} S_{3} l}{\rho \sigma(\varkappa-1)} .
$$

Будем численно искать минимум выражения (12) в зависимости от $\alpha$ с учетом (8)-(12), (17) при фиксированном масштабе турбулентности и заданном турбулентном потоке тепла, направленном вверх. Нетрудно убедиться в том, что для конвекции в земной атмосфере $(\gamma=7 / 5)$ минимальное производство энтропии достигается при $\alpha \sim 1.072, \varkappa \sim-4.556, \beta \sim-5.321$. Для Солнца, состоящего в основном из одноатомных газов, $\gamma=5 / 3$, и тогда решение задачи конвекции достигается при $\alpha \sim 1.125$, $\varkappa \sim-4.333, \beta \sim-5.083$.

\section{Перенос тепла в приземном слое атмосферы}

Рассмотрим стационарное неравновесное горизонтальное течение газа в условиях приземного слоя атмосферы. Тогда в уравнении (13) следует также учитывать генерацию энергии турбулентности действием сдвига скорости упорядоченного течения. Используя свойство постоянства турбулентного напряжения в приземном слое атмосферы [5]

$$
K_{v}\left|\frac{\partial v_{1}}{\partial x_{3}}\right|=u_{*}^{2},
$$

где константа $u_{*}$ - „скорость трения“, и выражения (15), (16), уравнение баланса энергии турбулентности для стационарного потока (13), приведем к уравнению относительно величины $X=b / u_{*}$

$$
\sigma X^{4}+\frac{E X}{\varkappa}-1=0
$$

где введен безразмерный параметр

$$
E=\frac{g_{3} J_{3} l}{\rho C_{p} u_{*}^{3}},
$$

характеризующий вклад работы силы Архимеда в поддержание энергии турбулентности по отношению к работе силы турбулентного трения. Используя выражения (9)-(11), (15), (18)-(20), численно определим - при каком значении величины $\alpha$, и заданном параметре $E$, скорость производства энтропии (12) достигает минимума. Решение поставленной задачи представлено в таблице. Результаты расчета указывают на то, что в зависимости от значения параметров в турбулентном потоке возможна самоорганизация нескольких типов стационарных неравновесных процессов:

1. При $E>3(\alpha>1, x<0)$ поток тепла направлен вверх, а поток массы - вниз. Атмосфера работает
Параметры стационарной конвекции в приземном слое атмосферы

\begin{tabular}{r|c|r|r|r|c}
\hline \multicolumn{1}{c|}{$E$} & $\alpha$ & \multicolumn{1}{c|}{$\varkappa$} & \multicolumn{1}{c|}{$\beta$} & \multicolumn{1}{c|}{$X$} & $\Gamma_{3}(\mathrm{~K} / \mathrm{km})$ \\
\hline 1000.00 & 1.07 & -4.56 & -5.32 & 16.84 & 1.83 \\
100.00 & 1.07 & -4.71 & -5.48 & 7.74 & 1.78 \\
10.00 & 1.06 & -5.67 & -6.42 & 3.54 & 1.52 \\
5.00 & 1.04 & -9.26 & -10.00 & 2.70 & 0.98 \\
4.00 & 1.02 & -15.67 & -16.40 & 2.44 & 0.60 \\
3.00 & 0.99 & 45.44 & 44.74 & 2.08 & -0.22 \\
2.00 & 0.38 & 1.65 & 1.37 & 0.81 & -7.10 \\
1.00 & -0.05 & 1.38 & 1.42 & 1.23 & -6.89 \\
0.50 & -0.62 & 1.25 & 1.69 & 1.65 & -5.78 \\
0.10 & -1.26 & 1.18 & 2.08 & 2.06 & -4.70 \\
0.05 & -1.33 & 1.17 & 2.12 & 2.11 & -4.60 \\
-0.05 & -1.47 & 1.16 & 2.21 & 2.21 & -4.41 \\
-0.10 & -1.54 & 1.16 & 2.26 & 2.26 & -4.32 \\
-0.50 & -1.95 & 1.14 & 2.53 & 2.62 & -3.87 \\
-1.00 & -2.25 & 1.12 & 2.73 & 2.99 & -3.58 \\
-10.00 & -2.82 & 1.10 & 3.12 & 5.85 & -3.13 \\
-100.00 & -2.87 & 1.10 & 3.15 & 12.54 & -3.10
\end{tabular}

как тепловая машина, при этом энергия турбулентности поддерживается за счет работы силы Архимеда. Предельному переходу $E \rightarrow \infty$ соответствует процесс свободной конвекции, рассмотренный выше.

2. При $0<\alpha<1, \quad x>1$ атмосфера работает как тепловая машина, но плотность газа при нагревании увеличивается. Потоки тепла и массы направлены вверх, а энергия турбулентности поддерживается за счет турбулентного трения.

3. При $\alpha<0$ атмосфера работает как тепловой насос, потоки тепла и массы направлены вверх, а энергия турбулентности поддерживается за счет турбулентного трения.

4. При $E<0$ атмосфера работает как тепловой насос, но потоки тепла и массы направлены вниз. Энергия турбулентности поддерживается за счет работы силы Архимеда.

5. Переход к режиму конвекции наступает при $E \sim 3$, при этом показатель политропы равен единице. В этой особой точке удельная теплоемкость уходит в бесконечность и мы приходим к случаю нулевого значения равновесного градиента температуры, соответствующего переходу (9) к закону Фурье.

\section{Заключение}

Дискуссия о значении „равновесного градиента температуры“, возникшая с момента создания молекулярнокинетической теории, продолжается до сего дня ([8]). Сложность решения этой задачи явилась следствием заблуждения о том, что эта величина является некой универсальной константой. На самом же деле турбулентный газ самоорганизуется в зависимости от внешних условий, реализуя соответствующий им термодинамический 
процесс. Изложенное в настоящей работе показывает, как принципы термодинамики неравновесных процессов позволяют установить удельную теплоемкость и иные термодинамические свойства газа в зависимости от значения турбулентного потока энтропии. Атмосфера может работать как тепловая машина, поддерживая энергию турбулентности за счет внутренней энергии, или как тепловой насос, нагнетая тепло от холодной области к горяей. В зависимости от значения удельной теплоемкости, плотность газа при нагревании может как увеличиться, так и уменьшиться.

Изложенный в данной работе подход позволил отказаться от априорного применения (весьма сомнительного) приближения Буссинеска к задаче конвекции в газах. Вместо этого при решении задач переноса в турбулентном газе следует начать с определения удельной теплоемкости, соответствующей протекаемому в нем термодинамическому процессу.

Найден ответ на „детский вопрос“ о природе конвекции, который можно сформулировать так: в поле силы тяжести (или иной массовой силы) при достаточно сильном неравномерном нагревании газа снизу и при масштабах процесса достаточно больших, чтобы могла развиться турбулентность, возникает такая самоорганизация материи, при которой показатель политропы станет меньшим единицы, а относительные флуктуации температуры и плотности - противоположными по знаку, что в результате работы силы Архимеда в атмосферной тепловой машине приведет к развитию конвекции.

\section{Список литературы}

[1] Ландау Л.Д., Лифиии, Е.М. Статистическая физика. Теоретическая физика. Т. 5. М.: Наука, 1968. 568 с.

[2] Румер Ю.Б., Рывкин М.Ш. Термодинамика, статистическая физика и кинетика. М.: Наука, 1977. 552 с.

[3] Ландау Л.Д., Лифшии, Е.М. Гидродинамика. Теоретическая физика. Т. 6. М.: Наука, 1988. 736 с.

[4] Кригель А.М. // ЖТФ. 2014. Т. 84. Вып. 11. С. 141-142.

[5] Монин А.С., Яглом А.М. Статистическая гидромеханика. Ч. 1. М.: Наука, 1965. 640 с.

[6] Кригель А.М. // Вестник ЛГУ. Сер. 7. 1991. Вып. 2. С. 107110.

[7] Пригожин И. Введение в термодинамику необратимых процессов. М.: ИИЛ, 1960. 128 с.

[8] Равновесный градиент температуры. Л.: Гидрометеоиздат, 1967. 